\title{
Linhong Wang, China CDC's Chief Expert of Chronic and Non-Communicable Disease Control
}

\author{
Peter Hao ${ }^{1, \alpha} ;$ Yu Chen ${ }^{2, x} ;$ Zhenjun $\mathrm{Li}^{1}$; Jingjing $\mathrm{Xi}^{1, * ;} ;$ Feng Tan ${ }^{1, *}$
}

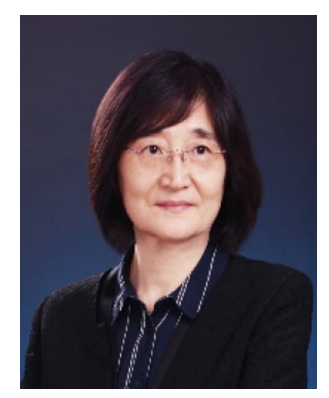

Linhong Wang is the Chief Expert of Chronic and Non-Communicable Disease (NCD) Control of China CDC and is a professional with over 30 years of experience in clinical and preventive medical science. Her specialties and professions span multiple fields with extraordinary contributions to public health.

Wang graduated from Beijing Medical University in 1983 with her first job out of university as an obstetrician and gynecologist in the Affiliated Hospital of the University. Under the influence and guidance of her predecessors, she expressed strong interests in maternal and children healthcare with a focus on the health of the larger population in the 1990s. In 1994, Wang pursued further studies in public health at the Centre for Environment and Population Health of Griffith University in Australia, where her knowledge in public health and technical skills in research became more in-depth and solid.

In 2002, she joined and created the National Center for Women and Children's Health of China CDC as the Deputy Director. During her tenure, Wang was engaged in conducting several studies and programs in women's health, reproductive health, and public health. Her research pursuits include but are not limited to the socioeconomic factors analysis of maternal mortality in rural China and its reduction, management of maternal health, and technical capacity training and control in high risk populations.

In 2002, Wang initiated a program for the prevention of mother-to-child transmission (PMTCT) of HIV in China. In this program, she served as the supervisor for a group of pioneers implementing prevention and control mechanisms by formulating policy and clinical treatment guidelines. She also led the group to create assessments of the effects of the intervention and cost-benefit evaluation in China. Wang also conducted a study for National Tenth Five-Year Plan of the Ministry of Science and Technology of China on the PMTCT of HIV transmission mechanism, which was a large-scale epidemiological survey of mother-to-child transmission of AIDS in China and required multidisciplinary research based on molecular virology, serology, and immunology to reveal the prevalence of maternal HIV/AIDS and the mother-to-child transmission mechanism. The results of this study have been used to tailor an approach to improving the system in China, which largely reduced the number of children infected with HIV/AIDS.

She has also been involved with the prevention of women's diseases, such as cervical cancer and breast cancer, with a focus on evaluating the effects of prevention and screening strategies. For instance, she has led the Chinese Breast Cancer Cohort Study (CBCCS) in the Precision Medical Research, which is one of the National Key Research and Development Programs. This study aimed to establish a standardized and sharable breast cancerspecific cohort to precisely identify etiology and to appropriately prevent and treat breast cancer, and this study has provided an open platform and reliable research database to improve the quality of follow ups by integrating existing resources.

As chronic diseases rose to wider public health concern, Wang shifted her academic focus to chronic and noncommunicable diseases in 2011 when she joined the National Center for Chronic and Non-Communicable Disease Control and Prevention of China CDC as a Director. In this role, Wang led and joined a series of key projects in chronic diseases and NCDs including China's surveillance and monitoring of the main NCDs and their high risk behaviors, the comprehensive management of the main NCDs of diabetes and hypertension, geographical variation analysis, disease burden research, nationwide investigations on chronic obstructive pulmonary disease, osteoporosis, mental health, elderly health in China, injury prevention, etc.

As a member of national consultation committee of Chinese National Health Commission and many 
international agencies, Wang is involved in national policy, work-plan development, and technical guideline development for relevant programs for NCDs and community health.

Wang is a member of Academic Commission of China CDC. She was also appointed as an Adjunct Professor in the Centre for Environment and Population Health of Griffith University in Australia and the School of Public Health at Peking University in China. Wang also actively participates in various professional and academic associations including: member of the Chinese Preventive Medicine Association (CPMA); Chairperson of the Women's Health Care Branch of CPMA; member of the Expert Commission of Disease Control and Prevention of China's NHC; Chief of Branch of Non-Communicable Disease Big Data Alliance, Chinese Health Information, and Medical Big Data Association.

She has published over 300 academic theses in various international and domestic academic journals and has been awarded multiple times. She has also edited more than 30 scholarly monographs as the Chief Editor.

Her hands-on academic and administrative experience enables Wang to develop insightful views on full life cycle health by saying: "From maternal health, birth safety, child and adolescent protection, to reproductive age women's health care, menopausal and elderly people's health, on to chronic and non-communicable disease prevention and control, my career speaks volumes for full life cycle health". As China CDC's Chief Expert of Chronic and NonCommunicable Disease Control, Linhong Wang continues to lead the country's response to diseases affecting full life cycle health.

doi: $10.46234 / \mathrm{ccdcw} 2020.189$

\# Corresponding authors: Jingjing Xi, xijj@chinacdc.cn; Feng Tan, tanfeng@chinacdc.cn.

\footnotetext{
${ }^{1}$ Chinese Center for Disease Control and Prevention, Beijing, China; ${ }^{2}$ National Institute of Environmental Health, Chinese Center for Disease Control and Prevention, Beijing, China.
}

\& Joint first authors.

Submitted: August 17, 2020; Accepted: August 24, 2020 